\title{
Silver Nanoparticles with Different Thiol Functionalization: An Opposite Optical Behaviour in Presence of $\mathrm{Hg}$ (II)
}

\author{
Luca Burratti1,a ${ }^{\text {* }}$, Iole Venditti2,b, Chiara Battocchio ${ }^{2, c}$, S. Casciardi ${ }^{3, d}$, \\ Paolo Prosposito ${ }^{1, \mathrm{e}}$ \\ ${ }^{1}$ Department of Industrial Engineering and INSTM, University of Rome Tor Vergata, Via del \\ Politecnico 1, Rome, 00133, Italy \\ ${ }^{2}$ Department of Sciences, Roma Tre University of Rome, Via della Vasca Navale 79, 00146 \\ Rome, Italy \\ ${ }^{3}$ National Institute for Insurance against Accidents at Work (INAIL), Department of Occupational \\ and Environmental Medicine, Epidemiology and Hygiene, 00078 Monte Porzio Catone, Italy \\ aluca.burratti@uniroma2.it, biole.venditti@uniroma3.it, 'chiara.battocchio@uniroma3.it, \\ ds.casciardi@inail.it, epaolo.prosposito@uniroma2.it
}

Keywords : Metal Nanomaterials, Silver Nanoparticles, Localized Surface Plasmon Resonance (L-SPR), Optical Sensors, Hg(II) Ions Detection

\begin{abstract}
We synthesized two different functionalized silver nanoparticles (AgNPs) in water, starting from silver nitrate, as Ag(I) ions precursor, and sodium borohydride, as reduction agent. The first system was capped with sodium 3-mercapto-1- propansulfonate (3MPS), while L-Cysteine and citrate stabilized the other system. We characterized both systems by UV-Vis absorption spectroscopy and transmission electron microscopy (TEM). We tested their optical response to several heavy metal ions monitoring the Localized Surface Plasmon Resonance (LSPR) band. In particular, these two systems have an opposite optical behaviour in presence of $\mathrm{Hg}(\mathrm{II})$ ions as contaminants. In the case of AgNPs-L-Cysteine/citrate, the plasmonic band shifted to higher wavelengths affording a linear behaviour and LOD, in the range from 1 to $7.5 \mathrm{ppm}$ and $600 \mathrm{ppb}$, respectively; whereas, the AgNPS-3MPS peak shifted to lower wavelengths with a linear range from 0 to $5 \mathrm{ppm}$ and a LOD of $240 \mathrm{ppb}$ for $\mathrm{Hg}$ (II). A preliminary hypothesis about the interaction mechanism between AgNPs and $\mathrm{Hg}$ (II) ions is discussed.
\end{abstract}

\section{Introduction}

Micro and nano-materials, display unique physical and chemical properties with respect to the same materials in bulk structure. These particular features are widely exploited in numerous fields, such as energy, optoelectronics, biomedicine and sensors[1-9]. In the field of sensors, optical properties can be based on reflectivity, photoluminescence emission and absorption of micro/nano-systems. When certain contaminants, such as small molecules, volatile organic compounds (VOCs), pesticides or heavy metal ions (HMIs) interact with the optical device, they are able to change the optical signal on which the sensor is based. In general, the variation of the signal can regard its intensity, its energy or its shape. These changes are related to the different nature of the interaction between the optical device and the external stimulus.

Among sensors based on light reflection, photonic crystals (PCs) show a Bragg-like diffraction in the visible range as a consequence of a peak in the reflectance spectrum[10-12]. The porous structure of PCs allows the permeation of contaminants between the spheres and a 
change of dielectric function of the whole device occurs causing a shift of the reflectivity band. Sensors based on PCs have been employed for the detection of several hazardous compound such us VOCs[13-16].

Although inorganic quantum dots (QDs), graphene oxide quantum dots (GOQDs) or metal nanoclusters (MNCs), have different chemical structures and are obtained with different synthetic strategies[17-22], they are linked by a common feature: the photoluminescence emission (PL). Also, in these cases, photoemitting material have been used to detect the presence of various species of pollutants[23-28]. The interaction mechanism would explain the opposite behaviour in the PL intensity change[29].

Among the sensors based on a change in optical absorption[30-32], metal nanoparticles (MNPs) show a typical absorption peak in the UV-Vis range, due to the phenomenon of Localized-Surface Plasmon Resonance (LSPR)[33]. To prevent the aggregation of these MNPs, during the chemical synthesis, a stabilizing molecule, which is also a species sensitive to the presence of the pollutants, is introduced. Colloidal solutions of MNPs show a specific colour depending on the type of noble metal (gold, silver or cupper), on the size and shape of the NPs[34], thus a shift of the plasmonic band produces a change of colour of the solution, which can be appreciated by naked eye (colorimetric sensors)[35-38]. The study and the development of optical sensors should be encouraged for the simplicity of the synthesis, the fast response to the external stimulus and the ease of data interpretation.

In this work, two different AgNPs systems functionalized with hydrophilic capping agents were synthesized, using citric acid (Cit) and L-Cysteine (L-Cys) for the first Ag-nanosystem and 3-mercapto-1-propanesulfonate (3MPS) for the second one. We characterized both systems, by Dynamic Light Scattering (DLS) and Transmission Electron Microscopy (TEM) analysis confirming nanosized dimensions. AgNPs- 3MPS and AgNPs-L-Cys/Cit were tested as plasmonic sensor for heavy metal detection in water, showing a good response for $\mathrm{Hg}$ (II) ions in both cases, but with an opposite behaviour. A hypothesis about the interaction mechanism between AgNPs and Hg(II) ions were discussed.

\section{Experimental section Chemical reagents}

Silver nitrate $\left(\mathrm{AgNO}_{3}\right)$, Sodium borohydride $\left(\mathrm{NaBH}_{4}\right)$, Sodium 3-mercapto-1propanesulfonate (3MPS), Sodium citrate $\left(\mathrm{Na}_{3} \mathrm{C}_{6} \mathrm{H}_{5} \mathrm{O}_{7}\right.$, Cit), L-Cysteine $\left(\mathrm{C}_{3} \mathrm{H}_{7} \mathrm{NO}_{2} \mathrm{~S}\right.$, L-Cys) have been purchased from Sigma-Aldrich. For the sensing tests we used the following metallic salt: $\mathrm{NaAsO}_{2}, \mathrm{NaHAsO}_{4} * 7 \mathrm{H}_{2} \mathrm{O}, \mathrm{Ca}\left(\mathrm{ClO}_{4}\right)_{2}, \mathrm{Cd}\left(\mathrm{NO}_{3}\right)_{2}, \mathrm{CoCl}_{2} * 6 \mathrm{H}_{2} \mathrm{O}, \mathrm{CrCl}_{3} * 6 \mathrm{H}_{2} \mathrm{O}$, $\mathrm{Cu}\left(\mathrm{NO}_{3}\right)_{2}, \mathrm{FeCl}_{3} * 6 \mathrm{H}_{2} \mathrm{O}, \mathrm{Hg}\left(\mathrm{NO}_{3}\right)_{2} * \mathrm{H}_{2} \mathrm{O}, \mathrm{KClO}_{4}, \mathrm{Mg}\left(\mathrm{ClO}_{4}\right)_{2}$,

$\mathrm{NaClO}_{4}, \mathrm{NdCl}_{3} * 6 \mathrm{H}_{2} \mathrm{O}, \mathrm{NiCl}_{2} * 6 \mathrm{H}_{2} \mathrm{O}, \mathrm{Pb}\left(\mathrm{NO}_{3}\right)_{2}, \mathrm{Zn}\left(\mathrm{NO}_{3}\right)_{2} * 6 \mathrm{H}_{2} \mathrm{O}$ purchased from SigmaAldrich. All chemical reagents have been used as received without any further process and all solutions have been prepared with deionized water (resistivity $18.2 \mathrm{M} \Omega \mathrm{cm}$ at $25^{\circ} \mathrm{C}$ ) obtained from Millipore Milli-Q water purification system.

\section{Apparatus}

Optical characterizations of bare AgNPs systems and contamination tests have been performed by Perkin-Elmer Lambda 19 UV/Vis/NIR spectrophotometer, in the range from $300 \mathrm{~nm}$ to 700 nm. The morphological characterization of the AgNPs has been accomplished with a Transmission Electron Microscope (TEM). The experimental apparatus is a FEI TECNAI 12 G2 $(120 \mathrm{KeV})$ equipped with an energy filter (GATAN GIF model) and a Peltier cooled SSC (slow scan charged coupled device) multiscan camera (794 IF model). 


\section{Synthesis of AgNPs}

The AgNPs stabilized by 3MPS were prepared by a wet reduction of $\mathrm{AgNO}_{3}$ with $\mathrm{NaBH}_{4}$. The detailed synthesis is reported in our previous works [39,40]. Briefly, the $\mathrm{AgNO}_{3}$ water solution is added dropwise to $\mathrm{NaBH}_{4}$ solution under vigorous stirring at the temperature of $3^{\circ} \mathrm{C}$; 3MPS was subsequently added and it capped the silver nanoparticles. After the synthesis is completed, the solution is stored at $\mathrm{T}=4^{\circ} \mathrm{C}$ before the analysis.

The AgNPs stabilized with citrate and L-Cysteine were prepared and characterized in analogy to literature reports [41-43]. Sodium citrate, L-Cys and $\mathrm{AgNO}_{3}$ were dissolved separately in distilled water and then the three solutions were mixed under magnetic stirring. After degassing the mixture with Argon for 10 minutes, a $\mathrm{NaBH}_{4}$ solution were added and allowed to react at room temperature for 2 hours. The obtained brown solution was purified by centrifugation, collecting the AgNPs in the precipitate and resuspended in water (13000 rpm, 10 min, 2 times with deionized water).

\section{Results and discussion}

Fig.1 shows a TEM image of the AgNPs-3MPS system: the particles have a mean diameter of about $4 \mathrm{~nm}$. The dimensional characterizations obtained by TEM analysis for both systems are listed in Table 1

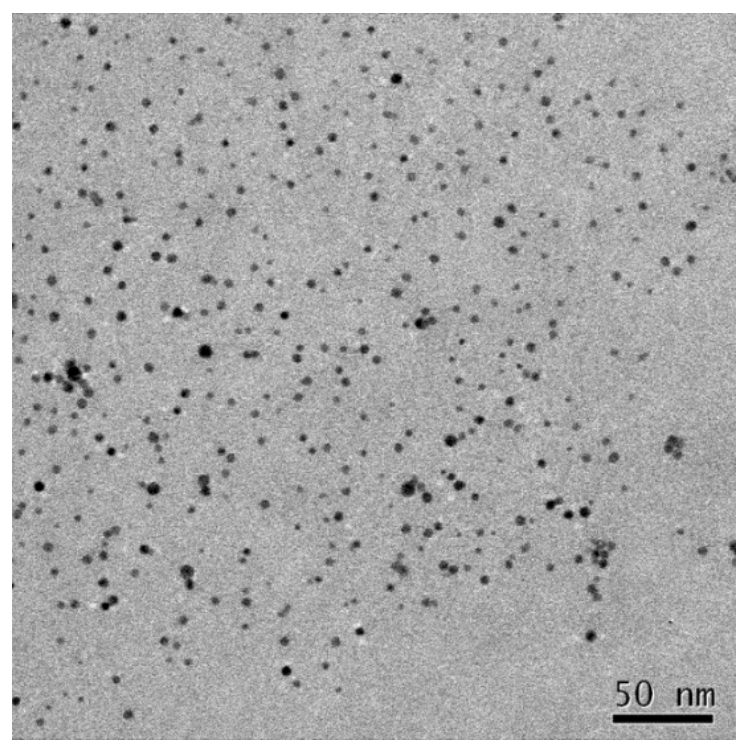

Fig. 1. TEM image of the AgNPs-3MPS system.

Fig. 2 shows the optical absorption in the UV-Vis range for both AgNPs systems. The solid black line represents the AgNPs-3MPS solution; the plasmon band absorption has the maximum centred at $396 \mathrm{~nm}$ and a Full Width at Half Maximum (FWHM) of $74 \mathrm{~nm}$. The green dashed curve is the absorption of the reference solution of AgNPs- L-Cys/Cit, in this case the maximum of the peak is at $401 \mathrm{~nm}$ and the FWHM is $102 \mathrm{~nm}$. 
Table 1. TEM size comparison.

\begin{tabular}{|c|c|c|}
\hline System & Size by TEM & REF. \\
\hline AgNPs-3MPS & $4.1 \pm 0.4 \mathrm{~nm}$ & {$[39]$} \\
\hline AgNPs-L-Cys/Cit & $5 \pm 2 \mathrm{~nm}$ & {$[43]$} \\
\hline
\end{tabular}

When both systems were contaminated with 5ppm of $\mathrm{Hg}(\mathrm{II})$ ions showed an opposite behaviour, the LSPR band of AgNPs-3MPS system shifted from about 400 to $350 \mathrm{~nm}$ (blue solid line), whereas for the AgNPs-L-Cys/Cit from 400 to around $430 \mathrm{~nm}$ (red dashed curve). Measuring the effect of different $\mathrm{Hg}(\mathrm{II})$ concentrations with step of $1 \mathrm{ppm}$, we obtained a linear behaviour from 0 to $5 \mathrm{ppm}$ and a limit of detection (LOD, 3 $\sigma$ ) of $240 \mathrm{ppb}$ for the AgNPs-3MPS system. Similarly, for the other silver nanoparticles system, we obtained a linear behaviour between 1 to $7.5 \mathrm{ppm}$ and we estimated a LOD of $600 \mathrm{ppb}$. We tested the optical response of both systems in presence of several metal ions checking the shape and energy of the LSPR at the concentration of $5 \mathrm{ppm}$. For some of them we did not find any difference in the respective LSPRs, for some others we found a similar behaviour, namely a shift of the LSPR band to longer wavelengths (red shift). The optical behaviour of the surface plasmon resonance band for the two silver systems in presence of the different heavy metal ions at the concentration of $5 \mathrm{ppm}$ is reported in Table 2. In the table, a positive value represents a red shift, while a negative value is referred to a blue shift. The AgNPs-3MPS system presented a low selectivity, showing a similar optical response towards $\mathrm{Cd}(\mathrm{II}), \mathrm{Co}$ (II), $\mathrm{Cu}(\mathrm{II}), \mathrm{Mg}(\mathrm{II})$ and $\mathrm{Ni}(\mathrm{II})$ (red shift), but evidencing in the case of $\mathrm{Hg}$ (II) an opposite behaviour with respect to the others contaminants (blue, more marked shift). Furthermore, the AgNPS-LCys/Cit was more selective with respect to the other system, since it responded with a red shift to $\mathrm{Cr}(\mathrm{III}), \mathrm{Cu}(\mathrm{II})$ and $\mathrm{Hg}(\mathrm{II})$ even if it is less sensitive. However, as already outlined, the opposite shift of the two systems in presence of mercury ions, makes this combined optical sensor really selective for that specific contaminant. A preliminary hypothesis about the different interaction mechanism for $\mathrm{Hg}(\mathrm{II})$ of the two systems, that can justify the opposite behaviour, is discussed in the next section.

\section{A preliminary hypothesis of the opposite optical behaviour in presence of $\mathbf{H g}$ (II)}

Some preliminary considerations about the coverage level of the capping agents for the two AgNPs systems has to be done. The AgNPs-3MPS system has a capping agent amount that is about 10 times lower than the $\mathrm{AgNO}_{3}$ content, which could mean the 3MPS molecules cover only partially the AgNPs surface. The low coverage level allows the $\mathrm{Hg}$ (II) ions to easily reach the NPs surface and to react with it, forming an amalgam with the external Ag atoms[44,45]. 


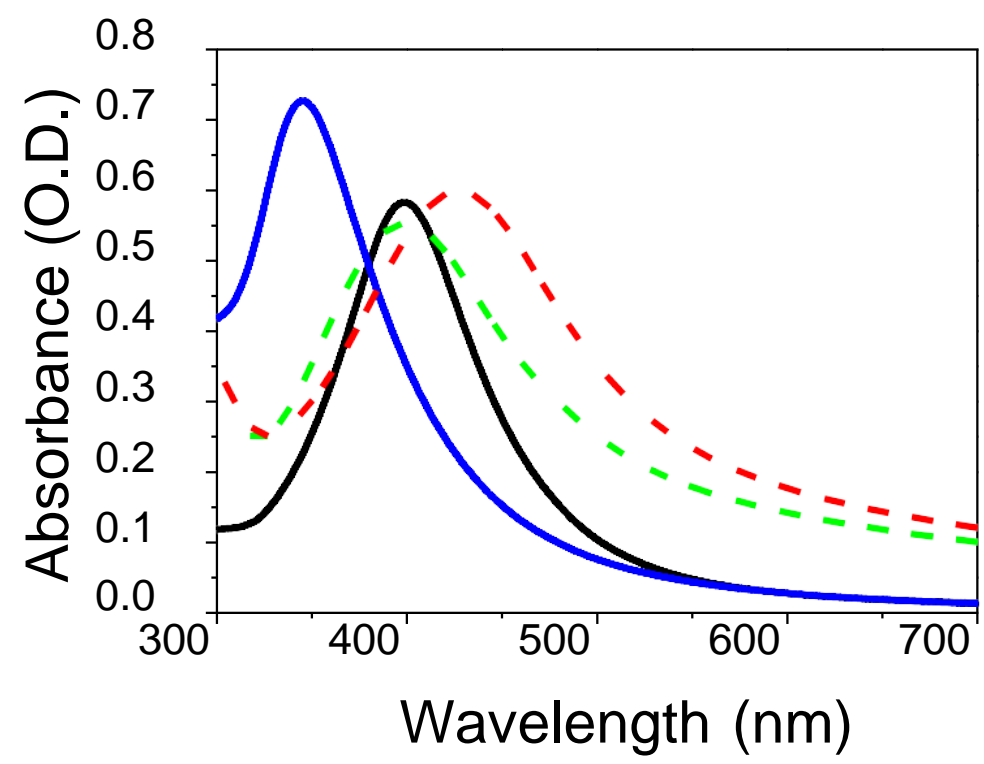

Fig. 2. UV-Vis absorption spectra of both AgNPs systems without and with 5ppm of $\mathrm{Hg}(\mathrm{II})$. AgNPs-3MPS as reference and with 5ppm of Hg(II) contamination are the solid black and the solid blue curve, respectively. AgNPs-L-Cys/Cit as reference and with 5ppm of Hg(II) contamination are the dashed green and the dashed red curve, respectively.

Table 2. heavy metal ions tested with both systems.

\begin{tabular}{|c|c|c|}
\hline & \multicolumn{2}{|c|}{ Ag nano-system } \\
\hline Metal ions at 5 ppm & NPs-3MPS & NPs-L-Cys/Cit \\
\hline As(III) & 1 & 1 \\
\hline As(V) & 1 & 1 \\
\hline $\mathrm{Ca}(\mathrm{II})$ & 1 & 1 \\
\hline $\mathrm{Cd}(\mathrm{II})$ & $+11 \mathrm{~nm}$ & 1 \\
\hline $\mathrm{Co}(\mathrm{II})$ & $+17 \mathrm{~nm}$ & 1 \\
\hline Cr(III) & 1 & $+8 \mathrm{~nm}$ \\
\hline $\mathrm{Cu}(\mathrm{II})$ & $+6 \mathrm{~nm}$ & $+6 \mathrm{~nm}$ \\
\hline $\mathrm{Fe}(\mathrm{III})$ & 1 & 1 \\
\hline $\mathrm{Hg}(\mathrm{II})$ & $-44 n m$ & $+26 \mathrm{~nm}$ \\
\hline $\mathrm{K}(\mathrm{I})$ & 1 & 1 \\
\hline $\mathrm{Mg}(\mathrm{II})$ & $+6 \mathrm{~nm}$ & 1 \\
\hline $\mathrm{Na}(\mathrm{II})$ & 1 & 1 \\
\hline $\mathrm{Nd}(\mathrm{III})$ & 1 & 1 \\
\hline $\mathrm{Ni}(\mathrm{II})$ & $+19 \mathrm{~nm}$ & 1 \\
\hline $\mathrm{Pb}(\mathrm{II})$ & 1 & 1 \\
\hline $\mathrm{Zn}(\mathrm{II})$ & 1 & 1 \\
\hline
\end{tabular}

The mechanism could be explained on the basis of the electrochemical differences of $\mathrm{Ag}(\mathrm{I})$ and $\mathrm{Hg}(\mathrm{II})$ ions. The standard reduction potential for $\mathrm{Ag}$ is $+0.80 \mathrm{~V}\left(\mathrm{Ag}(\mathrm{I})+\mathrm{e}^{-}=\operatorname{Ag}(0)\right)$ whereas for $\mathrm{Hg}(\mathrm{II})$ it is $+0.85 \mathrm{~V}$ ( $\mathrm{Hg}(\mathrm{II})+2 \mathrm{e}^{-}=\mathrm{Hg}(0)$ ) and according to the electrochemical series, metals with a higher reduction potential act as better oxidising agents[46,47]. Therefore, 
$\mathrm{Hg}$ (II) ions will reduce at the surface of AgNPs, while Ag atoms will oxidize, giving origin at the amalgam. This mechanism could explain the blue shift of LSPR band of AgNPs-3MPS.

In the other case, the two stabilizers (citrate and L-Cysteine) are about 14 times higher than the $\mathrm{Ag}(\mathrm{I})$ ions, thus the coverage of AgNPs surface is probably complete. The stability of AgNPs colloidal solutions is due to the electrostatic repulsions of functional groups of capping agents, indeed, L-Cys and Cit molecules with their carboxylate (-COO') groups prevent the aggregation of AgNPs in solution. When the AgNPs-L-Cys/Cit solution is in presence of $\mathrm{Hg}$ (II) ions, the latter cannot reach the bare surface of NPs. This favours the aggregation of the particles, and as consequence, we observe a red shift of the optical absorption and a broadening of the LSPR band.

\section{Conclusions}

In this study, we synthesized two different functionalized AgNPs in water, with a narrow size dispersion and a good stability over the time. We tested their optical response to several heavy metal ions monitoring the LSPR band before and after adding contaminated water. An opposite optical behaviour for the two systems in presence of $\mathrm{Hg}$ (II) ions have been measured. In the case of AgNPs-L-Cysteine/citrate, the plasmonic band shifted to higher wavelengths while in the case of AgNPS-3MPS shifted to lower wavelengths. This behaviour offers a very good selectivity in the detection of $\mathrm{Hg}(\mathrm{II})$ in water with a LOD of about $600 \mathrm{ppb}$. The opposite behaviour can be related to the different coverage level of NPs surface. For 3MPS Ag system, the capping agent amount is not enough to cover completely the NPs, thus the $\mathrm{Hg}$ (II) ions interact directly with the Ag external atoms, forming an amalgam which give rise to the blue shift in the absorption spectrum. For the other nano-system, where the coverage level is enough to completely shield the NPs surface, the $\mathrm{Hg}(\mathrm{II})$ ions interact with the particle surface, promoting the aggregation of AgNPs and as a consequence a red shift of the absorption spectrum was detected.

\section{Acknowledgement}

This research was partially funded by Regione Lazio, through Progetto di ricerca 85- 201715125, according to L.R.13/08 and by the University of Rome Tor Vergata in the framework of "GHOST" project, within "Mission Sustainability" program (D.R. 2817/2016), grant number (CUP): E86C18000450005. The Grant of Excellence Departments, MIUR (ARTICOLO 1, COMMI 314 - 337 LEGGE 232/2016), is gratefully acknowledged by authors of Roma Tre University.

\section{References}

[1] X. Cao, C. Tan, X. Zhang, W. Zhao, H. Zhang, Solution-Processed Two- Dimensional Metal Dichalcogenide-Based Nanomaterials for Energy Storage and Conversion, Adv. Mater. 28 (2016) 6167-6196. https://doi.org/10.1002/adma.201504833

[2] P. Prosposito, L. D’Amico, M. Casalboni, N. Motta, Periodic arrangement of monodispersed gold nanoparticles for high performance polymeric solar cells, in: 2015 IEEE 15th Int. Conf. Nanotechnol., IEEE, 2015: pp. 378-380. https://doi.org/10.1109/NANO.2015.7389005

[3] F. Xia, T. Mueller, Y. Lin, A. Valdes-Garcia, P. Avouris, Ultrafast graphene photodetector, Nat. Nanotechnol. 4 (2009) 839-843. https://doi.org/10.1038/nnano.2009.292 
[4] S.I. Valyansky, E.K. Naimi, L. V. Kozhitov, Functional 2D nanomaterials for optoelectronics based on langmuir bacteriorhodopsin films, Mod. Electron. Mater. 2 (2016) 7984. https://doi.org/10.1016/j.moem.2016.12.007

[5] S. Priyadarsini, S. Mohanty, S. Mukherjee, S. Basu, M. Mishra, Graphene and graphene oxide as nanomaterials for medicine and biology application, J. Nanostructure Chem. 8 (2018) 123-137. https://doi.org/10.1007/s40097-018-0265-6

[6] J.J. Giner-Casares, M. Henriksen-Lacey, M. Coronado-Puchau, L.M. Liz- Marzán, Inorganic nanoparticles for biomedicine: where materials scientists meet medical research, Mater. Today. 19 (2016) 19-28. https://doi.org/10.1016/j.mattod.2015.07.004

[7] M. Etienne, A. Goux, E. Sibottier, A. Walcarius, Oriented Mesoporous Organosilica Films on Electrode: A New Class of Nanomaterials for Sensing, J. Nanosci. Nanotechnol. 9 (2009) 2398-2406. https://doi.org/10.1166/jnn.2009.SE39

[8] P.K. Kannan, D.J. Late, H. Morgan, C.S. Rout, Recent developments in 2D layered inorganic nanomaterials for sensing, Nanoscale. 7 (2015) 13293-13312. https://doi.org/10.1039/C5NR03633J

[9] R. De Angelis, I. Venditti, I. Fratoddi, F. De Matteis, P. Prosposito, I. Cacciotti, L. D’Amico, F. Nanni, A. Yadav, M. Casalboni, M. V. Russo, From nanospheres to microribbons: Selfassembled Eosin Y doped PMMA nanoparticles as photonic crystals, J. Colloid Interface Sci. 414 (2014) 24-32. https://doi.org/10.1016/j.jcis.2013.09.045

[10]J. Hou, H. Zhang, Q. Yang, M. Li, L. Jiang, Y. Song, Hydrophilic- Hydrophobic Patterned Molecularly Imprinted Photonic Crystal Sensors for High-Sensitive Colorimetric Detection of Tetracycline, Small. 11 (2015) 2738-2742. https://doi.org/10.1002/smll.201403640

[11]Z. Cai, N.L. Smith, J.-T. Zhang, S.A. Asher, Two-Dimensional Photonic Crystal Chemical and Biomolecular Sensors, Anal. Chem. 87 (2015) 5013- 5025.

https://doi.org/10.1021/ac504679n

[12] Y. Zhang, Y. Zhao, R. Lv, A review for optical sensors based on photonic crystal cavities, Sensors Actuators A Phys. 233 (2015) 374-389. https://doi.org/10.1016/j.sna.2015.07.025

[13]C. Dispenza, M.A. Sabatino, S. Alessi, G. Spadaro, L. D’Acquisto, R. Pernice, G. Adamo, S. Stivala, A. Parisi, P. Livreri, A.C. Busacca, Hydrogel films engineered in a mesoscopically ordered structure and responsive to ethanol vapors, React. Funct. Polym. 79 (2014) 68-76. https://doi.org/10.1016/j.reactfunctpolym.2014.03.016

[14]L. Burratti, F. De Matteis, M. Casalboni, R. Francini, R. Pizzoferrato, P. Prosposito, Polystyrene photonic crystals as optical sensors for volatile organic compounds, Mater. Chem. Phys. 212 (2018) 274-281. https://doi.org/10.1016/j.matchemphys.2018.03.039

[15]L. Burratti, M. Casalboni, F. De Matteis, R. Pizzoferrato, P. Prosposito, Polystyrene opals responsive to methanol vapors, Materials (Basel). 11 (2018).

https://doi.org/10.3390/ma11091547

[16] M. Qin, M. Sun, R. Bai, Y. Mao, X. Qian, D. Sikka, Y. Zhao, H.J. Qi, Z. Suo, $\mathrm{X}$. He, Bioinspired Hydrogel Interferometer for Adaptive Coloration and Chemical Sensing, Adv. Mater. 30 (2018) 1800468. https://doi.org/10.1002/adma.201800468 
[17] J. Owen, L. Brus, Chemical Synthesis and Luminescence Applications of Colloidal Semiconductor Quantum Dots, J. Am. Chem. Soc. 139 (2017) 10939-10943. https://doi.org/10.1021/jacs.7b05267

[18]K.J. Nordell, E.M. Boatman, G.C. Lisensky, A Safer, Easier, Faster Synthesis for CdSe Quantum Dot Nanocrystals, J. Chem. Educ. 82 (2005) 1697. https://doi.org/10.1021/ed082p1697

[19]F. Liu, M.-H. Jang, H.D. Ha, J.-H. Kim, Y.-H. Cho, T.S. Seo, Facile Synthetic Method for Pristine Graphene Quantum Dots and Graphene Oxide Quantum Dots: Origin of Blue and Green Luminescence, Adv. Mater. 25 (2013) 3657-3662. https://doi.org/10.1002/adma.201300233

[20]Q. Lu, C. Wu, D. Liu, H. Wang, W. Su, H. Li, Y. Zhang, S. Yao, A facile and simple method for synthesis of graphene oxide quantum dots from black carbon, Green Chem. 19 (2017) 900-904. https://doi.org/10.1039/C6GC03092K

[21] L. Burratti, E. Ciotta, E. Bolli, M. Casalboni, F. De Matteis, R. Francini, S. Casciardi, P. Prosposito., Synthesis of fluorescent silver nanoclusters with potential application for heavy metal ions detection in water, in AIP Conference Proceedings; (2019): p. 020007. https://doi.org/10.1063/1.5123568

[22]L. Burratti, E. Bolli, M. Casalboni, F. de Matteis, F. Mochi, R. Francini, S. Casciardi, P. Prosposito, Synthesis of Fluorescent Ag Nanoclusters for Sensing and Imaging Applications, Mater. Sci. Forum. 941 (2018) 2243-2248. https://doi.org/10.4028/www.scientific.net/MSF.941.2243

[23]A.C. Vinayaka, S. Basheer, M.S. Thakur, Bioconjugation of CdTe quantum dot for the detection of 2,4-dichlorophenoxyacetic acid by competitive fluoroimmunoassay based biosensor, Biosens. Bioelectron. 24 (2009) 1615-1620. https://doi.org/10.1016/j.bios.2008.08.042

[24]R. De Angelis, L. D’Amico, M. Casalboni, F. Hatami, W.T. Masselink, P. Prosposito, Photoluminescence sensitivity to methanol vapours of surface InP quantum dot: Effect of dot size and coverage, Sensors Actuators, B Chem. 189 (2013) 113-117.

https://doi.org/10.1016/j.snb.2013.01.057

[25] M. Frasco, N. Chaniotakis, Semiconductor Quantum Dots in Chemical Sensors and Biosensors, Sensors. 9 (2009) 7266-7286. https://doi.org/10.3390/s90907266

[26]A. Ananthanarayanan, X. Wang, P. Routh, B. Sana, S. Lim, D.-H. Kim, K.-H. Lim, J. Li, P. Chen, Facile Synthesis of Graphene Quantum Dots from 3D Graphene and their Application for Fe 3+ Sensing, Adv. Funct. Mater. 24 (2014) 3021-3026.

https://doi.org/10.1002/adfm.201303441

[27]E. Ciotta, P. Prosposito, P. Tagliatesta, C. Lorecchio, L. Stella, S. Kaciulis, P. Soltani, E. Placidi, R. Pizzoferrato, Discriminating between different heavy metal ions with fullerenederived nanoparticles, Sensors (Switzerland). 18 (2018) 1-15. https://doi.org/10.3390/s18051496 [28] J.X. Dong, Z.F. Gao, Y. Zhang, B.L. Li, N.B. Li, H.Q. Luo, A selective and sensitive optical sensor for dissolved ammonia detection via agglomeration of fluorescent Ag nanoclusters and temperature gradient headspace single drop microextraction, Biosens. Bioelectron. 91 (2017) 155-161. https://doi.org/10.1016/j.bios.2016.11.062 
[29]A.T. Afaneh, G. Schreckenbach, Fluorescence Enhancement/Quenching Based on Metal Orbital Control: Computational Studies of a 6-Thienyllumazine- Based Mercury Sensor, J. Phys. Chem. A. 119 (2015) 8106-8116. https://doi.org/10.1021/acs.jpca.5b04691

[30]S. O’Keeffe, C. Fitzpatrick, E. Lewis, An optical fibre based ultra violet and visible absorption spectroscopy system for ozone concentration monitoring, Sensors Actuators B Chem. 125 (2007) 372-378. https://doi.org/10.1016/j.snb.2007.02.023

[31]H.-A. Ho, M. Béra-Abérem, M. Leclerc, Optical Sensors Based on Hybrid DNA/Conjugated Polymer Complexes, Chem. - A Eur. J. 11 (2005) 1718- 1724.

https://doi.org/10.1002/chem.200400537

[32] J. Wang, Y. Chang, W.B. Wu, P. Zhang, S.Q. Lie, C.Z. Huang, Label-free and selective sensing of uric acid with gold nanoclusters as optical probe, Talanta. 152 (2016) 314-320. https://doi.org/10.1016/j.talanta.2016.01.018

[33] K.A. Willets, R.P. Van Duyne, Localized Surface Plasmon Resonance Spectroscopy and Sensing, Annu. Rev. Phys. Chem. 58 (2007) 267-297. https://doi.org/10.1146/annurev.physchem.58.032806.104607

[34]K.M. Mayer, J.H. Hafner, Localized Surface Plasmon Resonance Sensors, Chem. Rev. 111 (2011) 3828-3857. https://doi.org/10.1021/cr100313v

[35]J. V. Rohit, J.N. Solanki, S.K. Kailasa, Surface modification of silver nanoparticles with dopamine dithiocarbamate for selective colorimetric sensing of mancozeb in environmental samples, Sensors Actuators, B Chem. 200 (2014) 219-226.

https://doi.org/10.1016/j.snb.2014.04.043

[36]D. Li, Y. Dong, B. Li, Y. Wu, K. Wang, S. Zhang, Colorimetric sensor array with unmodified noble metal nanoparticles for naked-eye detection of proteins and bacteria, Analyst. 140 (2015) 7672-7677. https://doi.org/10.1039/c5an01267h

[37]A. Jeevika, D.R. Shankaran, Functionalized silver nanoparticles probe for visual colorimetric sensing of mercury, Mater. Res. Bull. 83 (2016) 48-55.

https://doi.org/10.1016/j.materresbull.2016.05.029

[38] J.Y. Cheon, W.H. Park, Green synthesis of silver nanoparticles stabilized with musselinspired protein and colorimetric sensing of lead(II) and copper(II) ions, Int. J. Mol. Sci. 17 (2016). https://doi.org/10.3390/ijms17122006

[39]P. Prosposito, F. Mochi, E. Ciotta, M. Casalboni, F. De Matteis, I. Venditti, L. Fontana, G. Testa, I. Fratoddi, Hydrophilic silver nanoparticles with tunable optical properties: Application for the detection of heavy metals in water, Beilstein J. Nanotechnol. 7 (2016) 1654-1661. https://doi.org/10.3762/bjnano.7.157

[40]F. Mochi, L. Burratti, I. Fratoddi, I. Venditti, C. Battocchio, L. Carlini, G. Iucci, M. Casalboni, F. De Matteis, S. Casciardi, S. Nappini, I. Pis, P. Prosposito, Plasmonic Sensor Based on Interaction between Silver Nanoparticles and Ni2+ or Co2+ in Water, Nanomaterials. 8 (2018) 488. https://doi.org/10.3390/nano8070488

[41]A. Majzik, L. Fülöp, E. Csapó, F. Bogár, T. Martinek, B. Penke, G. Bíró, I. Dékány, Functionalization of gold nanoparticles with amino acid, $\beta$-amyloid peptides and fragment, 
Colloids Surfaces B Biointerfaces. 81 (2010) 235-241.

https://doi.org/10.1016/j.colsurfb.2010.07.011

[42] I. Venditti, G. Testa, F. Sciubba, L. Carlini, F. Porcaro, C. Meneghini, S. Mobilio, C. Battocchio, I. Fratoddi, Hydrophilic Metal Nanoparticles Functionalized by 2-

Diethylaminoethanethiol: A Close Look at the Metal- Ligand Interaction and Interface Chemical Structure, J. Phys. Chem. C. 121 (2017) 8002-8013. https://doi.org/10.1021/acs.jpcc.7b01424

[43] Prosposito, Burratti, Bellingeri, Protano, Faleri, Corsi, Battocchio, Iucci, Tortora, Secchi, Franchi, Venditti, Bifunctionalized Silver Nanoparticles as Hg2+ Plasmonic Sensor in Water: Synthesis, Characterizations, and Ecosafety, Nanomaterials. 9 (2019) 1353. https://doi.org/10.3390/nano9101353

[44]L. Li, L. Gui, W. Li, A colorimetric silver nanoparticle-based assay for Hg(II) using lysine as a particle-linking reagent, Microchim. Acta. 182 (2015) 1977-1981.

https://doi.org/10.1007/s00604-015-1536-2

[45] P.K. Sarkar, A. Halder, N. Polley, S.K. Pal, Development of Highly Selective and Efficient Prototype Sensor for Potential Application in Environmental Mercury Pollution Monitoring, Water, Air, Soil Pollut. 228 (2017) 314. https://doi.org/10.1007/s11270-017-3479-1

[46] G. V Ramesh, T.P. Radhakrishnan, A Universal Sensor for Mercury (Hg, Hg I , Hg II ) Based on Silver Nanoparticle-Embedded Polymer Thin Film, ACS Appl. Mater. Interfaces. 3 (2011) 988-994. https://doi.org/10.1021/am200023w

[47]S.S. Ravi, L.R. Christena, N. SaiSubramanian, S.P. Anthony, Green synthesized silver nanoparticles for selective colorimetric sensing of $\mathrm{Hg} 2+$ in aqueous solution at wide $\mathrm{pH}$ range, Analyst. 138 (2013) 4370. https://doi.org/10.1039/c3an00320e 